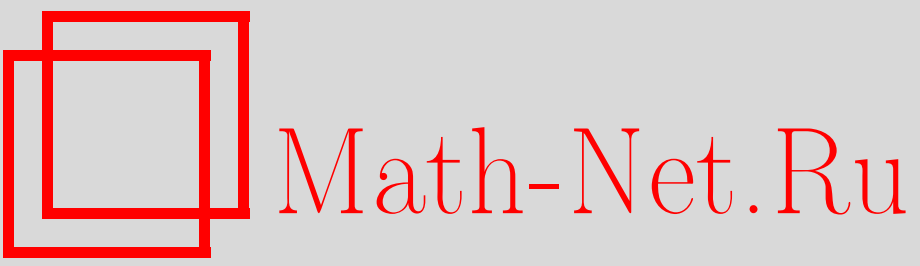

С. П. Суетин, Об интерполяционных свойствах диагональных аппроксимаций Паде эллиптических функций, УМН, 2004, том 59, выпуск 4, 201-202

DOI: https://doi.org/10.4213/rm767

Использование Общероссийского математического портала Math-Net.Ru подразумевает, что вы прочитали и согласны с пользовательским соглашением

http://www.mathnet.ru/rus/agreement

Параметры загрузки:

IP : 3.81 .55 .215

26 апреля 2023 г., 15:58:06 


\title{
ОБ ИНТЕРПОЛЯЦИОННЫХ СВОЙСТВАХ ДИАГОНАЛЬНЫХ АППРОКСИМАЦИЙ ПАДЕ ЭЛЛИПТИЧЕСКИХ ФУНКЦИЙ
}

\author{
С. П. Суетин
}

1. Пусть $f=\sum_{k=0}^{\infty} c_{k} / z^{k+1}-$ сходящийся в окрестности бесконечно удаленной точки $z=\infty$ степенной ряд, $\left\{f_{n}\right\}_{n \in \mathbb{N}}$ - последовательность его диагональных аппроксимаций Паде: $f_{n}=P_{n} / Q_{n}$, где $P_{n}, Q_{n} \in \mathbb{C}[z], \operatorname{deg} P_{n}, \operatorname{deg} Q_{n} \leqslant n, Q_{n} \not \equiv 0$ и $R_{n}(z):=\left(Q_{n} f-P_{n}\right)(z)=$ $O\left(1 / z^{n+1}\right)$ при $z \rightarrow \infty, R_{n}$ - функция остатка. Наиболее общий резултат о сходимости диагональных аппроксимаций Паде - теорема Шталя [1], из которой, в частности, следует, что если ряд $f$ представляет алгебраическую функиию, то последовательность $\left\{f_{n}\right\}_{n \in \mathbb{N}}$ сходится по емкости внутри области $D=\overline{\mathbb{C}} \backslash S$, где $S=S(f)$ - компакт минимальной емкости [2], [3], соответствующий выбранной рядом $f$ ветви алгебраической функции: $f$ продолжается из окрестности точки $z=\infty$ в дополнение к $S$ как мероморфная (т.е. однозначная аналитическая) функция, а из всех компактов, обладающих таким свойством, $S$ имеет наименьшую емкость. При этом (см. [4]-[7]) в проколотой области $D^{\prime}=D \backslash\{$ полюсы $f\}$, где продолженная функция голоморфна, полюсы рационалшных дробей $f_{n}$ могут иметь пределшные точки. Принято считать [4]-[7], что именно наличие у $f_{n}$ при $n \in \Lambda$ таких полюсов, компактно принадлежащих $D^{\prime}$, и является препятствием к равномерной сходимости последовательности $\left\{f_{n}\right\}_{n \in \mathbb{N}}$ внутри $D^{\prime}$. Для некоторых классов гиперэллиптических функций удалось показать [8], [9], что динамика этих полюсов подчиняется определенной закономерности. Известно [4]-[7], что таким полюсам сопутствуют близкие нули $f_{n}$. Тем самым, уместно говорить о парах полюс-нуль, называемшіх дефектами [4; гл. $2, \S 2.2]$. Так как асимптотически нули и полюсы в этих парах взаимно сокращаются, то принято считать [4], что их появление носит случайньй характер и никак не связано с природой функции $f$. Такой подход к этому явлению отразился и на соответствующей терминологии: подобные полюсы и нули аппроксимаций стали называть "ложньми". Особенно негативно сказьвается их появление при использовании аппроксимаций Паде в приложениях [4], в связи с чем при $n \in \Lambda$ и сами аппроксимации Паде $f_{n}$ предлагается считать дефектными и исключать из рассмотрения [4; гл. $2, \S 2.3]$ или использовать процедуры так называемого "очищения" от ложных полюсов [6].

2. В [10] Дюма исследовал вопрос об асимптотическом поведении последовательности $\left\{d_{n}\right\}_{n \in \mathbb{N}}$ для эллиптической функции вида

$$
d(z)=\sqrt{\left(z-e_{1}\right) \cdots\left(z-e_{4}\right)}-z^{2}+\left(e_{1}+\cdots+e_{4}\right) z / 2,
$$

где точки $e_{1}, \ldots, e_{4} \in \mathbb{C}$ попарно различны и выбрана та ветвь корня, главный член которой в окрестности точки $z=\infty$ равен $z^{2}$; тем самым $d \in \mathscr{H}(\infty)$. Дюма, в частности, показал, что в "общем положении" множество полюсов дробей $d_{n}$ всюду плотно в $\overline{\mathbb{C}}$.

В [11] (см. также [12]) результат Дюма был распространен на некоторый класс эллиптических функций. При этом было отмечено $[11 ; \S 1$, п. 2] следующее явление: при каждом $n$, принадлежащем некоторой подпоследовательности $\Lambda$, всегда существует точка $\beta=\beta_{n} \in D=\overline{\mathbb{C}} \backslash S$, для которой $d_{n}\left(\beta_{n}\right)=\widetilde{d}\left(\beta_{n}\right)$, где $\widetilde{d}(z)=-\sqrt{\left(z-e_{1}\right) \cdots\left(z-e_{4}\right)}-z^{2}+\left(e_{1}+\cdots+e_{4}\right) z / 2$ - другая ветвь эллиптической функции (1) в $D$. Таким образом, для каждого $n$ из некоторой подпоследовательности $\Lambda$ диагональные аппроксимации Паде $d_{n}$ интерполируют в некоторой точке области $D$ другую ветвь функции $d$. При этом, в случае общего положения такие точки "неправильной" интерполящии $\left\{\beta_{n}\right\}_{n \in \Lambda}$ всюду плотны в $\overline{\mathbb{C}}$.

В настоящей заметке приводятся более общие результаты в этом направлении.

3. Рассмотрим класс эллиптических функций вида

$$
f(z)=w(z) / q(z)-r(z)
$$

где $w(z)=\sqrt{\left(z-e_{1}\right) \cdots\left(z-e_{4}\right)}, q \in \mathbb{C}[z]-$ произвольный полином, который на $S$ может иметь лишь простые нули в точках $e_{1}, \ldots, e_{4}$, а $r \in \mathbb{C}(z)$ есть сумма главных частей мероморфоной в области $D=\overline{\mathbb{C}} \backslash S$ функции $w(z) / q(z)$; тем самым, $f \in \mathscr{H}(D)$ (при $q(z) \equiv 1$ получаем функцию

Работа выполнена при финансовой поддержке фонда INTAS (грант № 03-51-6637) и программы поддержки ведущих научных школ России (грант № НШ-1551.2003.1). 
Дюма (1)). Пусть $\left\{f_{n}\right\}_{n \in \mathbb{N}}-$ последовательность диагональных аппроксимаций Паде функции $f$, $\widetilde{f}(z)=-w(z) / q(z)-r(z)-$ другая ветвь функции $(2)$ в $D$.

Теорема 1. В “общем положении" для произвольной функции $f$ вида (2) имеем: $\mathbb{N}=\Lambda_{1} \sqcup \Lambda_{2} \sqcup \Lambda_{3}$, әде плотности последовательностей $\Lambda_{1}=\Lambda_{1}(f)$ и $\Lambda_{2}=\Lambda_{2}(f)$ равнь $1 / 2$, при каждом $n \in \Lambda_{1}$ существует точка $\alpha_{n} \in D$, а при каждом $n \in \Lambda_{2}$ точка $\beta_{n} \in D$ такие чтол:

1) $f_{n}\left(\alpha_{n}\right)=f\left(\alpha_{n}\right)$ npu $n \in \Lambda_{1} u\left\{\alpha_{n}\right\}_{n \in \Lambda_{1}}^{\prime}=\overline{\mathbb{C}}$;

2) $f_{n}\left(\beta_{n}\right)=\widetilde{f}\left(\beta_{n}\right)$ nрu $n \in \Lambda_{2} u\left\{\beta_{n}\right\}_{n \in \Lambda_{2}}^{\prime}=\overline{\mathbb{C}}$.

(При этом $f_{n} \rightarrow f$ равномерно внутри $D$ при $n \rightarrow \infty, n \in \Lambda_{1}$.)

В условиях теоремы 1 для произвольного достаточно малого $\varepsilon>0$ через $\Lambda_{2}^{\varepsilon}$ обозначим множество $n \in \Lambda_{2}$ таких, что $\beta_{n} \in D^{\varepsilon}$, где $D^{\varepsilon}=\overline{\mathbb{C}} \backslash S^{\varepsilon}$, а $S^{\varepsilon}-2 \varepsilon$-окрестность множества $S$.

Теорема 2. Пусть в условиях теоремы $1 \varepsilon>0$ - произвольное достаточно малое число, $K_{n}^{\varepsilon}=\left\{z \in D:\left|z-\beta_{n}\right|<\varepsilon\right\}$ nри $n \in \Lambda_{2}^{\varepsilon}$. Тогда для всех достаточно больших $n \in \Lambda_{2}^{\varepsilon}$ имеем: $f_{n}\left(K_{n}^{\varepsilon}\right)=\overline{\mathbb{C}}$.

Таким образом, из теоремы 2 вытекает, что существование ложных полюсов и нулей диагональных аппроксимаций Паде $f_{n}$ функций вида (2) является непосредственным следствием наличия в области $D$ точек, в которых эти диагональные аппроксимации интерполируют другую ветвь $\widetilde{f}$ функции $f$ (и, тем самым, существование таких полюсов и нулей напрямую связано с природой аппроксимируемой функции). Естественно назвать такие точки ложными точками интерполяции для диагональных аппроксимаций Паде или просто ло жными точками для диагональных аппроксимаций Паде.

4. Приведем формальное определение для общего случая гиперэллиптических функций. Пусть $f(z)=r_{1}(z)+r_{2}(z) w(z)$, где $r_{1}, r_{2} \in \mathbb{C}(z), w(z)=\sqrt{\left(z-e_{1}\right) \cdots\left(z-e_{2 g+2}\right)}, g \in \mathbb{N}$, точки $e_{1}, \ldots, e_{2 g+2}$ попарно различны и выбрана такая ветвь корня, что $w(z) / z^{g+1} \rightarrow 1$ при $z \rightarrow \infty$. Область $D=\overline{\mathbb{C}} \backslash S$, где $S$ - компакт минимальной емкости, соответствующий функции $w(z), \widetilde{f}$ - другая ветвь гиперэллиптической функции $f$ в $D: \widetilde{f}(z)=r_{1}(z)-r_{2}(z) w(z)$.

ОПредЕЛЕниЕ. Для произволњного фиксированного $n \in \mathbb{N}$ точку $\beta=\beta_{n} \in D$ назовем ложной точкой для диагональной аппроксимации Паде $f_{n}$ гиперэллиптической функции $f$, если $r_{2}(\beta) \neq 0$ и $f_{n}(\beta)=\widetilde{f}(\beta)$.

Отметим, что впервые явно на это явление ложной интерполяции указано в работе Буслаева [13]: именно на этом свойстве $f_{n}$ основан его контрпример к известной гипотезе БейкераГаммеля-Уиллса (соответствующая функция Буслаева - гиперэллиптическая рода $g=2$; см. также [14]).

\section{СПИСОК ЛИТЕРАТУРЫ}

[1] H. Stahl // Constr. Approx. 1986. V. 2. P. 225-240; 241-251. [2] J. Nuttall // J. Approx. Theory. 1984. V. 42. P. 299-386. [3] H. Stahl // Complex Variables. 1985. V. 4. P. 311-324; 325-338; 339-354. [4] Дж. Бейкер, П. Грейвс-Моррис. Аппроксимации Паде. М.: Мир, 1986. [5] A. Baker George, Jr. // Acta Appl. Math. 2000. V. 61. № 1-3. P. 37-52. [6] H. Stahl // Ann. Fac. Sci. Toulouse. Math. (6). Spec. Iss. 1996. P. 121-193. [7] H. Stahl // Constr. Approx. 1997. V. 13. Р. 287-292. [8] E. А. Рахманов // Матем. сб. 1977. T. 104 (146). № 2. C. 271-291. [9] С. П. Суетин // Матем. сб. 2002. Т. 193. № 12. С. 81-114. [10] S. Dumas. Sur le développement des fonctions elliptiques en fractions continues. Thesis. Zürich, 1908. [11] C. П. Суетин // Матем. сб. 2003. Т. 194. № 12. С. 63-92. [12] С. П. Суетин // УМН. 2002. Т. 57. № 2. С. 199-200. [13] В. И. Буслаев // Матем. сб. 2002. Т. 193. №6. С. 25-38. [14] С. П. Суетин // УМН. 2003. T. 58. № 4. С. 159-160. 